\title{
Pattern of Bacteria and Its Susceptibility of Ventilator-Associated Pneumonia Patients in ICU at Dr. Hasan Sadikin General Hospital, Bandung
}

\author{
Achmad Faisal Jundi, ${ }^{1}$ Nurita Dian KSS, ${ }^{2}$ Yanti Mulyana $^{3}$ \\ ${ }^{1}$ Faculty of Medicine Universitas Padjadjaran, ${ }^{2}$ Department of Anesthesiology and Intensive \\ Care Faculty of Medicine Universitas Padjadjaran/Dr. Hasan Sadikin General Hospital Bandung, \\ ${ }^{3}$ Department of Microbiology and Parasitology, Faculty of Medicine Universitas Padjadjaran
}

\begin{abstract}
Background: Incidence of ventilator-associated pneumonia (VAP) is common in intensive care unit (ICU). This pneumonia, becomes important due to its association to increased length of hospital stay, increased costs, and increased mortality. Irrational administration of antibiotics can lead to development of bacterial resistance. The objective of this study was to describe the pattern of bacteria and the susceptibility of VAP patients.

Methods: A descriptive study was carried out to 10 medical records of VAP patients in the ICU at Dr. Hasan Sadikin General Hospital, Bandung in January-June 2014. Microbiological culture results and antibiotics susceptibility testing of patients' sputum were identified and the collected data were analyzed in form of tables and percentages by using a computer program.

Results: Out of 44 cultures taken from 10 patients with VAP, 43 cultures had microbiological findings. Gram negative bacteria were found in $90.6 \%$ cultures, the rests were Gram positive bacteria and fungi. The majority of findings were: Acinetobacter baumannii (41.9\%), Pseudomonas aeruginosa (18.6\%), and Klebsiella pneumoniae (16.9\%). The laboratory test result showed varied degrees of antimicrobial resistance to antibiotics.

Conclusions: Bacteria found in sputum cultures of ICU patient with VAP are mostly of Gram negative. The result of susceptibility testing of bacteria shows many of the bacteria have resistance to antibiotics in ICU at Dr. Hasan Sadikin General Hospital. [AMJ.2016;3(2):195-9]
\end{abstract}

Keywords: Bacteria pattern, Intensive Care Unit, Susceptibility, Ventilator-Associated Pneumonia

\section{Introduction}

Ventilator-associated pneumonia (VAP) is one of the most common infections found in the intensive-care unit (ICU). A study by Aly et al. ${ }^{1}$ in a hospital ICU, showed VAP as the most common nosocomial infection. This pneumonia, becomes important due to its association to increased length of hospital stay, increased costs, and increased mortality. ${ }^{2}$ Chung et al. ${ }^{3}$ explained that the most common bacteria causing pneumonia in hospitals were Acinetobacter spp., Pseudomonas aeruginosa, Staphylococcus aureus, and Klebsiella pneumoniae.

There is no "gold standard" method to determine accurately the cause of pneumonia in the ICU, so that selection and use of antibiotics therapy for its management is based on empirical use. ${ }^{4}$ Whereas development of bacterial resistance to antibiotics which have a multifactorial cause has shown to have high correlation to irrational use of antibiotics. ${ }^{5}$ The data which is not integrated in hospital has made the correct prescription of drugs becoming difficult and thus may lead to irrational use of drugs in management for VAP. The aim of this study was to identify the bacteria pattern of VAP patients in Dr. Hasan Sadikin General Hospital.

\section{Methods}

A descriptive study was carried out based on

Correspondence: Achmad Faisal Jundi, Faculty of Medicine, Universitas Padjadjaran, Jalan Raya Bandung-Sumedang Km.21, Jatinangor, Sumedang, Indonesia, Phone: +62 85715024740 Email: kennjashouto@gmail.com 
medical records of VAP patients in the ICU of Dr. Hasan Sadikin General Hospital, Bandung during the period January-June 2014. The study was conducted after obtaining approval from the Health Research Ethics Committee of the Faculty of Medicine, Universitas Padjadjaran and Dr. Hasan Sadikin General Hospital. The study population was all medical records of patients who suffered nonspecific pneumonia during their stay in the ICU, and the sample to be examined was taken based on total sampling.

The inclusion criterion of this study was the medical records of patients who had a diagnosis of VAP, either as primary diagnosis or additional diagnosis. The exclusion criteria were patients with respiratory infections other than VAP before admission to the ICU, and whose laboratory test results were neither complete nor available. After having determined the samples, it was required to gain access to the database of the Clinical Pathology Laboratory of Dr. Hasan Sadikin General Hospital, Bandung to observe data of microbiological culture results and antibiotics susceptibility testing of patients' sputum. Then, the collected data were analyzed in form of tables and percentages by using a computer program.

\section{Results}

There were 35 patients with unspecified pneumonia hospitalized in the ICU of Dr.
Hasan Sadikin General Hospital in the period January-June 2014. Among them, were 10 patients diagnosed as VAP. From those patients 44 sputum samples were taken for culture to be examined at different times, and in 43 were found microorganisms that later were cultured and tested for its susceptibility to antibiotics.

Most of the bacteria detected were gramnegative bacteria, namely Acinetobacter baumannii, followed by Pseudomonas aeruginosa and Klebsiella pneumoniae.

Furthermore, Achromobacter was known to be sensitive to Piperacillin-Tazobactam and Ceftazidime. Acinetobacter baumannii had $\geq$ $50 \%$ sensitivity to Amikacin, Cotrimoxazole, or Tigecycline. While, Burkholderia cepacia was found only fully sensitive to Cefepime. Enterobacter cloacae was found only sensitive to Tigecyclin. Klebsiellap neumoniae was fully sensitive to Ertapenem and Cefmetazone; and showed a slight degree of resistance to Amikacin, Meropenem, and Tigecyclin. Pseudomonas aeruginosa had the lowest resistance against Meropenem. Serratia marcescens was fully sensitive to the Fluoroquinolone class, Carbapenem class, Cefepime, and Tigecycline. While Staphylococci was only sensitive to Cotrimoxazole, Linezolid, Moxifloxacin, Tetracycline, Tigecycline, and Vancomycin (Table 2).

\section{Discussion}

Incidence of VAP is associated with the

Table 1 Bacterial Pattern of Sputum Cultures from 10 Patients Diagnosed with VAP

\begin{tabular}{lcc}
\hline \multicolumn{1}{c}{ Microorganisms Found } & $\begin{array}{c}\text { Number of Findings } \\
\text { (n=43) }\end{array}$ & Percentage (\%) \\
\hline Gram-negative Bacteria & 1 & 2.3 \\
Achromobacter & 18 & 41.9 \\
Acinetobacter baumannii & 1 & 2.3 \\
Burkholderia cepacia & 2 & 4.7 \\
Enterobacter cloacae & 7 & 16.9 \\
Klebsiella pneumoniae & 8 & 18.6 \\
Pseudomonas aeruginosa & 2 & 4.7 \\
Serratia marcescens & & \\
Gram-positive Bacteria & 1 & 2.3 \\
Staphylococcus aureus & 1 & 2.3 \\
Staphylococcus haemolyticus & & 4.7 \\
Others & 2 & \\
Candida sp. & & \\
\hline
\end{tabular}


Table 2 Pattern of Sensitivity of Microorganisms to Antibiotics (\%) of Sputum Cultures from Patients Diagnosed with VAP ( $n=43)$

\begin{tabular}{|c|c|c|c|c|c|c|c|c|c|c|}
\hline \multirow[b]{2}{*}{ Antibiotics } & \multicolumn{7}{|c|}{ Gram-negative Bacteria (90.6\%) } & \multicolumn{2}{|c|}{$\begin{array}{c}\text { Gram-positive } \\
\text { Bacteria (4.6\%) }\end{array}$} & \multirow{2}{*}{ 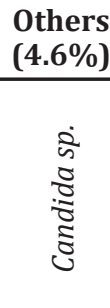 } \\
\hline & 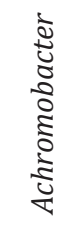 & 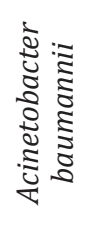 & 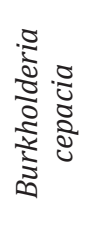 & 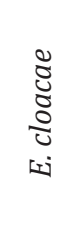 & 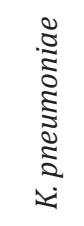 & 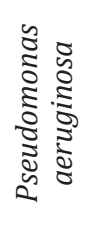 & 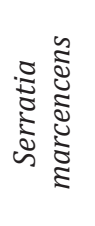 & 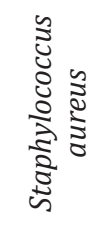 & 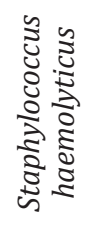 & \\
\hline \multicolumn{11}{|l|}{ Aminoglycoside } \\
\hline Amikacin & 0 & 66.7 & 0 & 0 & 71.4 & 37.5 & 75 & - & - & - \\
\hline Gentamycin & 0 & 19.4 & 0 & 0 & 28.6 & 25 & 0 & 0 & - & - \\
\hline Aztreonam & 0 & 0 & 0 & 0 & 28.6 & 31.3 & 0 & - & - & - \\
\hline \multicolumn{11}{|l|}{$\begin{array}{l}\beta \text {-lactam/ with } \\
\text { adjuvant }\end{array}$} \\
\hline Ampicillin-Sulbactam & 0 & 28 & 0 & 0 & 14.3 & 0 & 0 & 0 & 0 & - \\
\hline Oxacillin & - & - & - & - & - & - & - & - & 0 & - \\
\hline Piperacillin-Tazobactam & 100 & 0 & 0 & 0 & 35.7 & 25 & 50 & - & - & - \\
\hline Erythtromycin & - & - & - & - & - & - & - & 100 & 0 & - \\
\hline \multicolumn{11}{|l|}{ Fluoroquinolone } \\
\hline Ciprofloxacin & 0 & 0 & 0 & 0 & 42.9 & 12.5 & 100 & 0 & 0 & - \\
\hline Levofloxacin & 0 & 5.6 & 0 & 0 & 57.1 & 12.5 & 100 & 0 & 0 & - \\
\hline Moxifloxacin & - & - & - & - & - & - & - & - & 100 & - \\
\hline \multicolumn{11}{|l|}{ Carbapenem } \\
\hline Ertapenem & - & - & - & - & 100 & - & - & - & - & - \\
\hline Imipenem & - & - & - & - & - & - & - & 0 & 0 & - \\
\hline Meropenem & 50 & 5.6 & 0 & 0 & 71.4 & 75 & 100 & 0 & 0 & - \\
\hline Cotrimoxazole & 100 & 50 & 0 & 0 & 42.9 & 0 & 0 & 100 & 100 & - \\
\hline Linezolid & - & - & - & - & - & - & - & 100 & 100 & - \\
\hline \multicolumn{11}{|l|}{ Cephalosporin } \\
\hline Cefadroxil & - & - & 0 & - & - & - & - & - & - & - \\
\hline Cefazoline & 0 & 0 & 0 & 0 & 28.6 & 0 & 0 & 0 & 0 & - \\
\hline Cefepime & 0 & 0 & 100 & 0 & 28.6 & 25 & 100 & 0 & 0 & - \\
\hline Cefmetazone & - & - & - & - & 100 & 0 & - & - & - & - \\
\hline Cefoxitine & - & - & - & - & - & - & - & 0 & - & - \\
\hline Cefoperazone & - & - & 50 & - & - & - & 0 & 0 & - & - \\
\hline Cefotaxime & - & - & 0 & - & - & - & - & - & - & - \\
\hline Ceftazidime & 100 & 0 & 0 & 0 & 28.6 & 25 & 0 & 0 & 0 & - \\
\hline Ceftriaxone & 0 & 0 & 0 & 0 & 28.6 & 0 & 50 & 0 & 0 & - \\
\hline Cefuroxime & - & - & 0 & - & - & - & - & 0 & 0 & - \\
\hline Tetracycline & - & - & - & - & - & - & - & - & 100 & - \\
\hline Tigecycline & 50 & 63.9 & 50 & 100 & 85.7 & 0 & 100 & 100 & 100 & - \\
\hline Vancomycin & - & - & - & - & - & - & - & 100 & 100 & - \\
\hline
\end{tabular}


use of ventilator and or endotracheal tube, particularly within the ICU environment. The VAP occurs 48-72 hours or more after installation of ventilators or intubation procedures. ${ }^{6} \quad$ Adequate and immediate antibiotic therapy is essential in the management of VAP. Porzecanski et al. $^{7}$ explained about the adequate antibiotic therapy with the administration of at least one type of antibiotics in an appropriate dose, to sensitive organisms. Selection and the use of antibiotics in management of VAP should be based on the risk of developing drug resistance in microorganisms. ${ }^{8}$

Moreover, the minimum duration recommended for the treatment of VAP vary from 7-21 days depending on bacteria found which caused VAP. The de-escalation method is also recommended for an antibiotics therapy, by giving early broad spectrum followed by narrowing the spectrum or decreasing the dose once the laboratory result identifying the bacteria is known. Antibiotics need to be adjusted after bacterial culture and antibiotic susceptibility testing of specimens obtained from patients were revealed. The use of local antibiogram based on patterns of bacteria and its sensitivity to antibiotics is important in determining the initial selection of empiric therapy. ${ }^{6,9,10}$

This study found most of the bacteria that cause VAP in ICU of Dr. Hasan Sadikin General Hospital were Gram-negative bacteria (90.6\%), with the most common findings were Acinetobacter baumannii (41.9\%), Pseudomonas aeruginosa (18.6\%), and Klebsiella pneumoniae (16.9\%) (Table 1). This was in accordance with the study conducted by Aly et al. ${ }^{1}$ which showed the majority of microorganisms found in their research about nosocomial infections were Gramnegative bacteria (68\%), with most findings: Pseudomonas aeruginosa (17\%), Acinetobacter baumannii (13\%), Klebsiella spp. (11\%).

The results of this study also determined the resistance of bacteria through the results of antibiotics susceptibility tests in the Clinical Pathology Laboratory of Dr. Hasan Sadikin General Hospital, in the form of sensitivity patterns which are listed in Table 2. Amikacin, Tigecycline, Meropenem, and Levofloxacin showed lower percentages of bacterial resistance in susceptibility tests; therefore they were recommended to be considered as empirical antibiotics therapy in patients with clinical diagnosis of VAP. These findings, according to microbiology culture and antibiotics susceptibility tests, showed varied degrees of resistance to antibiotics. Due to this, it was important to perform microbiological examination and antibiotics susceptibility tests on every patient with VAP.

The limitations of this study were the small scales used and the short period of time it was performed; thus it was considered inaccurate to know about the real state of the case. The recommendations that can be proposed from this study is to consider a further study with a larger scale and more comprehensive, so it can properly document the present circumstances, especially in Dr. Hasan Sadikin General Hospital. Another limitation was the availability of data in hospitals, as well as neat and systematic documentation.

It can be concluded that microorganisms causing infection in patients with diagnosis of VAP in ICU of Dr. Hasan Sadikin General Hospital are mostly Gram-negative bacteria (90.6\%). Acinetobacter baumannii (41.9\%), Pseudomonas aeruginosa (18.6\%), and Klebsiella pneumoniae (16.9\%) are the most common microorganisms found.

\section{References}

1. Aly NY, Al-Mousa HH, Al Asar el SM. Nosocomial infections in a medicalsurgical intensive care unit. Med Princ Pract. 2008;17(5):373-7.

2. HunterJD.Ventilatorassociated pneumonia. Postgrad Med. 2006;82(965):172-8.

3. Chung DR, Song J-H, Kim SH, Thamlikitkul V, Huang S-G, Wang H, et al. High prevalence of multidrug-resistant nonfermenters in hospital-acquired Ppneumonia in Asia. Am J Respir Crit Care Med. 2011;184(12):140917.

4. Marino PL. The ICU book. 3rd ed. Philadelphia: Lippincott Williams \& Wilkins; 2007.

5. van Zanten ARH, Polderman KH. Rational use of antibiotics in the ICU: optimum efficacy for the lowest costs. In: Vincent $\mathrm{J}$-L, editor. Yearbook of intensive care and emergency medicine 2005. New York:Springer; 2005. p. 337-48.

6. Niederman M, Craven D. Guidelines for the management of adults with hospitalacquired, ventilator-associated, and healthcare-associated pneumonia. Am J Respir Crit Care Med. 2005;171(4):388416.

7. Porzecanski I, Bowton DL. Diagnosis and treatment of ventilator-associated pneumonia. Chest. 2006;130(2):597-604.

8. Garnacho-Montero J, Corcia-Palomo Y, 
Achmad Faisal Jundi, Nurita Dian KSS, Yanti Mulyana: Pattern of Bacteria and Its Susceptibility of Ventilator- 199 Associated Pneumonia Patients in ICU at Dr. Hasan Sadikin General Hospital, Bandung

Amaya-Villar R, Martin-Villen L. How to treat VAP due to MDR pathogens in ICU patients. BMC Infect Dis. 2014;14(1):135.

9. Charles MVP, Kali A, Easow JM, Joseph NM, Ravishankar M, Srinivasan S, et al. Ventilator-associated pneumonia.
Australas Med J. 2014;7(8):334-44.

10. Morrow BM, Argent AC, Jeena PM, Green RJ. Guideline for the diagnosis, prevention and treatment of paediatric ventilatorassociated pneumonia. S Afr Med J. 2009;99 (4 Pt 2):255-67 